\title{
Die Kommission Nachhaltigkeit@DRG stellt sich vor
}

Die Deutsche Röntgengesellschaft möchte das Thema Nachhaltigkeit stärker in der Radiologie und in der Fachgesellschaft selbst verankern und hat dafür die Kommission Nachhaltigkeit@DRG ins Leben gerufen. Auch hat die DRG verschiedene Maßnahmen für mehr Nachhaltigkeit entwickelt.

Aktuell wird der Begriff Nachhaltigkeit gesellschaftlich viel diskutiert, und auch im Gesundheitswesen finden diese Debatten vermehrt statt. Doch wie steht es eigentlich mit diesem Thema in der Radiologie und in der Deutschen Röntgengesellschaft? Die Deutsche Röntgengesellschaft hat sich ent-

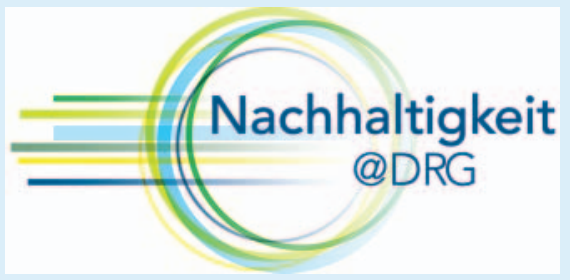

schlossen, den Weg der Radiologie hin zu mehr Nachhaltigkeit aktiv zu unterstützen und mitzugestalten und hat dafür die Kommission Nachhaltigkeit@DRG gegründet.

Die Kommission steht unter der Leitung von Dr. Kerstin Westphalen. Dr. Westphalen ist zudem Präsidentin des 103. Deutschen Rönt- genkongresses 2022, auf dem das Thema Nachhaltigkeit eines der zentralen Themen sein wird. Auf der Webseite der Kommission Nachhaltigkeit@DRG erfahren Sie mehr über die Kommission - siehe Infokasten.

Außerdem hat der Vorstand der DRG einen „10-Punkte-Plan für mehr Nachhaltigkeit@DRG“ verabschiedet, der verschiedene Maßnahmen enthält, um mehr Nachhaltigkeit in die DRG zu bringen.

Lernen Sie die Kommission Nachhaltigkeit@DRG kennen und besuchen Sie sie auf www.nachhaltigkeit.drg.de. 